Research Article:

\title{
The spa Typing and Characterization of Nasal Carriage Methicillin-resistant Staphylococcus aureus Isolates From Healthy Children
}

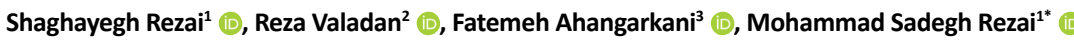

1. Pediatric Infectious Diseases Research Center, Mazandaran University of Medical Sciences, Mazandaran, Iran.

2. Molecular and Cell Biology Research Center, Department of Immunology, School of Medicine, Mazandaran University of Medical Sciences, Mazandaran, Iran.

3. Antimicrobial Resistance Research Center, Student Research Committee, Mazandaran University of Medical Sciences, Mazandaran, Iran

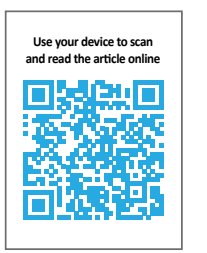

ditation Rezai S, Valadan R, Ahangarkani F, Sadegh Rezai M. The spa Typing and Characterization of Nasal Carriage Methicillinresistant Staphylococcus aureus Isolates From in Healthy Children. Journal of Pediatrics Review. 2020; 8(1):59-64. http://dx.doi. org/10.32598/jpr.8.1.59

dol $h$ htp://dx.doi.org/10.32598/jpr.8.1.59

Article info:

Received: 04 Aug 2019

First Revision: 08 Aug 2019

Accepted: 11 Aug 2019

Published: 01 Jan 2020

\section{Key Words:}

Methicillin-resistant

Staphylococcus aureus,

Molecular typing,

Children, Community-

acquired infections

\begin{abstract}
A B STRACT
Background: The Methicillin-resistant Staphylococcus aureus (MRSA) strains cause wide spectrum infections in hospitals and are responsible for the majority of the communityacquired infections. The emergence of community-acquired MRSA in patients without risk factors represents a high risk for public health.

Objectives: In this study, we aimed to molecular typing the nasal carriage MRSA strains harbored mecA gene isolated from healthy children living in the North of Iran and also to determine the pattern of vancomycin susceptibility in these isolates.

Methods: The MRSA strains were identified using standard microbiological and molecular procedures. The antibiotic susceptibility test was performed according to the clinical and laboratory standards institute. The MRSA strains were characterized by spa typing.

Results: In total, 14 different patterns of spa genes were obtained from MRSA isolates in this study, which included t790 (23.07\%), t2962 (15.38\%), t267 (15.38\%), t159 (7.69\%) and t701, t094, t1816, t11332, t937, t2135, t180, t160 [1 (3.84\%) isolate per each type]. The rate of resistant isolates to cefoxitin, , cefalotin, clindamycin, cefazolin, co-amoxiclav, co-trimoxazole, and cephalexin antibiotics were $69.23 \%, 100 \%, 46.15 \%, 96.15 \%, 100 \%, 3.48 \%$, and $100 \%$, respectively. In total, six (23.07\%) revealed vancomycin-intermediate resistant phenotype and five (19.23\%) isolates revealed vancomycin-resistant S. aureus phenotype.

Conclusions: This study showed the different variants of MRSA strains based on molecular typing, among healthy children. Continuous surveillance studies to monitor MRSA should be encouraged to gain a better understanding of the circulating MRSA strains and prevent community spread of this pathogen.
\end{abstract}




\section{Introduction}

taphylococcus aureus, as an important cause of community-acquired and nosocomial infections, has remained a significant health problem across the world (1-4). Methicillinresistant Staphylococcus aureus (MRSA) was first recognized in the early 1960s, shortly after the introduction of methicillin, a narrow spectrum semi-synthetic penicillin in clinical practice, through the acquisition of the mecA gene. This gene encodes for a modified form of penicillin-binding protein, which has a lower affinity for all beta-lactam antibiotics (3-5). The MRSA strains are responsible for a wide spectrum of clinical diseases, ranging from superficial infection of the skin and soft tissue to severe and life-threatening diseases such as pneumonia, sepsis, osteomyelitis, endocarditis, and toxic shock syndrome $(1,2)$.

After the emergence of MRSA in the clinical practice, in the late 1980s, vancomycin became the first choice for treatment of MRSA infections in the world. Shortly after that, S. aureus isolates with reduced susceptibility to teicoplanin, a structural relative of vancomycin, were reported in Europe in 1990. Subsequently, the emergence of Vancomycin-intermediate resistant (VISA) and Vancomycin-resistant S. aureus (VRSA) isolates were reported in 1997 and 2002, respectively. Since then, the increased rate of VRSA was reported from all over the world. The option for treatment of MRSA-induced infections and clinical symptoms are limited. Therefore, MRSA has remained a significant threat to human health globally (6-12).

Molecular typing of MRSA is an essential tool in the surveillance of healthcare-associated infections. Rapid identification of the strains will help control and prevention of MRSA, causing community-acquired or nosocomial infections. There are several molecular techniques for MRSA typing such as Multilocus Sequence Typing (MLST), Pulsedfield Gel Electrophoresis (PFGE), Staphylococcal Cassette Chromosome mec (SCCmec) typing and Staphylococcal Protein A (spa) typing (1). These methods are accurate and time-consuming. Thus, due to laborious character and difficulties in comparing data between laboratories and the requirement for inter-laboratory standardization, spa typing method, which is based on assessment of short sequence repeats of hypervariable $X$ region in the spa gene, exhibits excellent discriminatory power and has become a useful typing tool, with cheaper procedure, and standardized nomenclature (7).

Furthermore, spa typing allows data comparison between clinical laboratories at the international and na- tional levels $(13,14)$. The prevalence of different spa types among $S$. aureus isolates varies in different areas around the world (9). Although several studies have reported the spa typing and characterization of MRSA in patients in healthcare settings, this subject has not been investigated in healthy children in the North of Iran. The present study aimed at molecular typing via spa typing and characterization of nasal carriage methicillin-resistant Staphylococcus aureus isolates from healthy children living in the North of Iran.

\section{Methods}

\subsection{Isolation and identification of Methicillin-resistant S. aureus, vancomycin-intermediate resistant, and van- comycin-resistant $S$. aureus strains}

In this descriptive study, 26 non-duplicate MRSA isolates were recovered from healthy children aged 6-12 years living in the North of Iran. These isolates were identified as $S$. aureus methicillin-resistant, as we described previously (4). Identification of these isolates was performed according to the standard microbiological procedures (morphology, Gram stain, catalase test, coagulase test, mannitol salt agar fermentation, and molecular procedures). Antibiotic susceptibility test was performed by the Kirby-Bauer method according to the Clinical and Laboratory Standards Institute (CLSI) guideline and antibiotic disks, including cefoxitin, cefalotin, clindamycin, cefazolin, co-amoxiclav, co-trimoxazole, and cephalexin (15).

For detection of MRSA strains, oxacillin screen agar was used. Staphylococcus strains were cultured on Muller Hinton agar containing $4 \% \mathrm{NaCl}$, and six $\mathrm{mg} / \mathrm{L}$ oxacillin and then incubated for 24 hours $(4,12)$. Genomic DNA was extracted by the boiling method. Then for confirmation of MRSA strains, the isolates were subjected to Multiplex PCR assay to detect spa and mecA genes, and the previously described protocol was adjusted (4). For detection of VISA and VRSA isolates, the minimum inhibitory concentration of vancomycin by microdilution broth method according to CLSI standards was performed. VISA was defined when S. aureus strains exhibited increased resistance to vancomycin ( $\mathrm{MIC}=4-8 \mu \mathrm{g}$ / $\mathrm{mL}$ ), and VRSA as $S$. aureus isolates showed complete resistance to vancomycin (MIC $\geq 16 \mu \mathrm{g} / \mathrm{mL}$ ) $(15,16)$.

\subsection{The spa typing of MRSA strains}

Spa type is a repeated sequence comprising 24 repeated nucleotides (eight codons). To do typing, the short Sequence Repeats (SSR) of the polymorphic $X$ 
region of the protein A gene (spa) from MRSA isolates were amplified with the following primers: spa-1113F 5' - TAAAGACGATCCTTCGGTGAGC -3' and spa-1514R 5' -C AGCAGTAGTGCCGTTTGCTT -3'. PCR reactions were performed in $25 \mu \mathrm{L}$ final volumes containing $2 \mu \mathrm{L}$ of purified DNA, $0.5 \mu \mathrm{L}$ of each primer, $12.5 \mu \mathrm{L}$ of $2 \times$ Green PCR Master Mix and $8.5 \mu \mathrm{L}$ of distilled water. The PCR amplification conditions for spa primer were as follows: the initial denaturation at $94^{\circ} \mathrm{C}$ for five minutes, and next 30 cycles consisting of a denaturation step at $94^{\circ} \mathrm{C}$ for 30 seconds, annealing at $59^{\circ} \mathrm{C}$ for one minute, extension at $72^{\circ} \mathrm{C}$ for one minute, as well as a final extension step at $72^{\circ} \mathrm{C}$ for ten minutes, and storage at $4^{\circ} \mathrm{C}$ at the end. $S$. aureus ATCC 25923 contains spa gene and considered as the positive control.

Amplified products were directly sequenced for both strands using the PCR primers by Bioneer Company (Seoul, South Korea). Sequences were then checked for the quality and trimmed by CLC Genomics version 12.0.2 (Qiagen, Germany). The spa typing was per- formed by the module implicated inside the BioNumerics software version 7.6.3 (Applied Maths, Belgium). The sequences were submitted to Genbank under the accession number MK443456-MK443471. Finally, the type ability of this study was calculated as $87.1 \%$ with the method described previously (17).

\section{Results}

A total of 26 healthy boys with the Mean \pm SD age of $8.84 \pm 3.36$ year (age range: 6 to 12 year), nasal carrying MRSA, enrolled in this study. All MRSA strains isolated from these children contained the mecA gene. The rate of resistant isolates to cefoxitin, cefalotin, clindamycin, cefazolin, co-amoxiclav, co-trimoxazole, and cephalexin were $69.23 \%, 100 \%, 46.15 \%, 96.15 \%, 100 \%, 3.48 \%$, and $100 \%$, respectively. Table 1 presents the in vitro susceptibilities of vancomycin against MRSA isolates. Totally six (23.07\%) and five (19.23\%) isolates were VISA and VRSA, respectively.

Table 1. In vitro susceptibilities of vancomycin against Methicillin resistant Staphylococcus aureus isolates

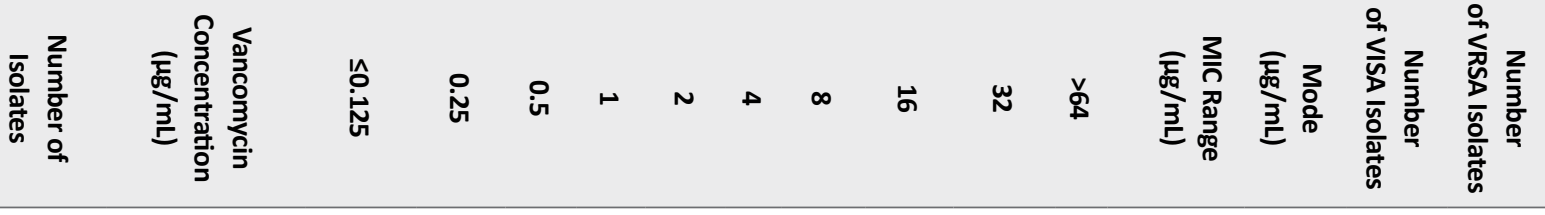

$$
\begin{aligned}
& 26 \begin{array}{c}
\begin{array}{c}
\text { Number of } \\
\text { isolates with } \\
\text { MIC }(\mu \mathrm{mg} / \mathrm{mL})
\end{array} \\
2
\end{array}
\end{aligned}
$$

VRSA: Vancomycin-resistant S. aureus; VISA: Vancomycin Intermediate Resistant S. aureus

Journal of Pediatrics Review

Table 2. Molecular characterization of MRSA strains with spa typing method

\begin{tabular}{|c|c|c|c|}
\hline No. & Spa Type & Repeat Succession & Number of Isolates \\
\hline 1 & $\mathrm{t} 790$ & $26-23-13-23-31-29-17-25-17-25-16-28$ & 6 \\
\hline 2 & $\mathrm{t} 267$ & $07-23-12-21-17-34-34-34-33-34$ & 4 \\
\hline 3 & t2962 & $26-23-13-23-25-28$ & 4 \\
\hline 4 & $\mathrm{t} 159$ & $14-44-13-12-17-17-23-18-17$ & 2 \\
\hline 5 & $\mathrm{t} 701$ & $11-10-21-17-34-24-34-22-25-25$ & 1 \\
\hline 6 & t094 & $07-23-12-34-34-12-12-23$ & 1 \\
\hline 7 & $\mathrm{t} 1816$ & $07-12-21-17-34-13-34-34-34-33-34$ & 1 \\
\hline 8 & $\mathrm{t} 11332$ & $04-21-12-41-486-17-12-12-17$ & 1 \\
\hline 9 & t937 & $08-16-34-24-34-34-17-17$ & 1 \\
\hline 10 & $\mathrm{t} 2135$ & $08-16-02-16-34-13-17-34-16$ & 1 \\
\hline 11 & $\mathrm{t} 180$ & $09-02-16-34-34-34-17-34-16-34$ & 1 \\
\hline 12 & $\mathrm{t} 160$ & $07-23-21-24-33-22-17$ & 1 \\
\hline 13 and 14 & $\begin{array}{l}\text { New types identified in this study, } \\
\text { and submitted in the spa server } \\
\text { (www.spaserver.ridom.de) }\end{array}$ & $\begin{array}{l}\text { 23-13-23-31-29-17-25-17-25-16-28 } \\
\text { And 08-16-02-16-02-31-25-17-17-24-24 }\end{array}$ & 2 \\
\hline
\end{tabular}

Journal of Pediatrics Review 
The typing of all isolates yielded 14 spa types as following: t790 (23.07\%), t2962 (15.38\%), t267 (15.38\%), t159 (7.69\%) and t701, t094, t1816, t11332, t937, t2135, t180, t160 (1 [3.84\%] isolate per each type) (Table 2). An interesting finding of this study was the emergence of two new spa types found with the Ridom Spa Server that was submitted in the server (www. spaserver.ridom.de).

\section{Discussion}

The MRSA strains produce various clinical manifestations in the hospitals and the community (18-22). This pathogen can adapt to a variety of conditions, and successful clones can result in epidemic or pandemic infections (7).

In this study, we characterized the MRSA strains isolated from the nose of healthy children. Although children have higher persistent carriage rates than adults, the rate of nasal carrying $S$. aureus varies depending on age, from approximately $45 \%$ during the first eight weeks to $21 \%$ by six months. Nevertheless, there is a transition from persistent carriage to intermittent or no carriage states during adolescence (18). Generally, nasal carriers of MRSA have an increased risk of acquiring infection with this pathogen. In our study, we found five MRSA strains harbored mecA gene, which were also VRSA by the phenotypical method. Our findings are alarming for the presence of vancomycin-resistant MRSA strains among healthy children, which should be addressed in future studies with larger sample sizes. Many Iranian laboratories do not follow a specific guideline for reporting and confirming VRSA, so the prevalence of VRSA strains is not known in our country. Askari et al. in a systematic review reported 24 VRSA isolates from Iran up to September 2012 (11).

Vancomycin is the primary antibiotic for the treatment of serious infections caused by MRSA strains. However, VRSA is defined as an isolate with a minimum inhibitory concentration of vancomycin greater than or equal to $16 \mu \mathrm{g} / \mathrm{mL}$ based on the determination by broth microdilution method and there are some reports regarding the genotype-negative phenotype-positive VRSA within resistance range $(13,16)$. Further studies are needed to advance identification of VISA and VRSA isolates, which in turn can be used to assess better the prevalence of these isolates, as well as to facilitate the development of optimal treatments (16). Although new therapies such as phage therapy have been suggested for the treatment of MRSA infection and this new therapeutic option has a promising future as an alternative or a supple- ment to antibiotic treatments of infection due to MRSA strains, antibiotic therapy is still the first choice (23-25).

Nowadays, typing and analysis of MRSA strains have become a routine procedure in many countries and are being used as a tool to show the relationship between strains and clones. Although many MRSA genotypes and clonal clusters have been identified in different geographic regions, certain gene clones are predominant in some regions (26-30). For example, in the current study and among 26 MRSA isolates, we found 14 different genotypes. Asadollahi et al. in a comprehensive review reported that The Netherlands has the most diverse range of spa types (34 types), followed by China (22 types), Germany (16 types), UK (15 types), Spain (11 types), Sweden (10 types), Iran (8 types), France (7 types), and Switzerland ( 6 types). In our study, we detected that the spa type t790 (in six isolates) was predominant, and t2962 (in four isolates), t267 (in four isolates), t159 (in two isolates) and t701, t094, t1816, t11332, t937, t2135, t180, t160 (in one isolate) were in the next lines.

Asadollahi et al. reported that the spa types t008 and t002 are the most frequently repeated spa types in 16 countries. The following most frequently repeated spa types were t037 (12 countries), t044 (11 countries), t084 (8 countries), t012 and t127 (7 countries), t041 ( 6 countries), and t019, t011, t034, t355, t189, t304 (5 countries) (7). In Asia, t030 was the dominant type located in China, while in Iran, this type reported as the fifth most common spa type (7).

Fourteen spa types among 26 MRSA strains of nasal carriage $S$. aureus are caused by different variants of the spa genes. Detection of the molecular characteristics of S. aureus isolates is important for distinguishing the relations between isolates, and consequently, the implementation of appropriate infection control measures. Conclusion of this study showed the different variants of MRSA strains among healthy Iranian children. Moreover, the characterized isolates with reduced sensitivity to vancomycin are of concern. Continuous surveillance studies to monitor MRSA strains and the health education in school should be encouraged to gain a better understanding of the circulating MRSA and to prevent community-spread of this pathogen.

\section{Ethical Considerations}

\section{Compliance with ethical guidelines}

In line with the principles of research ethics, written informed consent was obtained from the parents of the 
children. This study was approved by the Ethics Committee of Qaemshahr Branch, Azad University of Qaemshahr (378. ID Code: 10730548952006).

Funding

This research did not receive any specific grant from funding agencies in the public, commercial, or not-forprofit sectors.

\section{Authors' contributions}

All authors contributed in preparing this article.

\section{Conflicts of interest}

The authors declared no conflict of interest.

\section{Acknowledgements}

The authors would thank the personnel of Cellular and Molecular Research center and the staff of the Department of Medical Microbiology of Mazandaran University of Medical Sciences for their kind assistance.

\section{References}

1. Tong SY, Davis JS, Eichenberger E, Holland TL, Fowler VG Jr. Staphylococcus aureus infections: Epidemiology, pathophysiology, clinical manifestations, and management. Clinical Microbiology Reviews. 2015; 28(3):603-61. [DOI:10.1128/CMR.00134-14] [PMID] [PMCID]

2. Knox J, Uhlemann AC, Lowy FD. Staphylococcus aureus infections: Transmission within households and the community. Trends in Microbiology. 2015; 23(7):437-44. [DOI:10.1016/j.tim.2015.03.007] [PMID] [PMCID]

3. Strommenger B, Braulke C, Heuck D, Schmidt C, Pasemann B, Nubel U, et al. Spa typing of Staphylococcus aureus as a frontline tool in epidemiological typing. Journal of Clinical Microbiology. 2008; 46(2):574-81. [DOI:10.1128/ JCM.01599-07] [PMID] [PMCID]

4. Rezai S, Peyravii Ghadikolaii F, Ahanjan M, Valadan R, Ahangarkani $F$, et al. Prevalence of nasal carriage methicillin-resistant Staphylococcus aureus with meca gene among healthy primary school boys in North of Iran; A cross-sectional study. International Journal of Pediatrics. 2017; 5(12):6515-25.

5. Carvalho SP, Almeida JB, Andrade Y, Silva L, Oliveira AC, Nascimento FS, et al. Community-acquired methicillinresistant Staphylococcus aureus carrying SCCmec type IV and $\mathrm{V}$ isolated from healthy children attending public daycares in northeastern Brazil. The Brazilian Journal of Infectious Diseases. 2017; 21(4):464-7. [DOI:10.1016/j. bjid.2017.04.001] [PMID]

6. Deinhardt-Emmer S, Sachse S, Geraci J, Fischer C, Kwetkat A, Dawczynski K, et al. Virulence patterns of Staphylococcus aureus strains from nasopharyngeal colonization. The Journal of Hospital Infection. 2018; 100(3):309-15. [DOI:10.1016/j.jhin.2017.12.011] [PMID]

7. Asadollahi P, Farahani NN, Mirzaii M, Khoramrooz SS, van Belkum A, Asadollahi K, et al. Distribution of the most prevalent spa types among clinical isolates of methicillin-resistant and -susceptible Staphylococcus aureus around the world: A review. Frontiers in Microbiology. 2018; 9:163. [DOI:10.3389/fmicb.2018.00163] [PMID] [PMCID]

8. Adhikari R, Pant ND, Neupane S, Neupane M, Bhattarai R, Bhatta $S$, et al. Detection of methicillin resistant Staphylococcus aureus and determination of minimum inhibitory concentration of vancomycin for Staphylococcus aureus isolated from pus/wound swab samples of the patients attending a tertiary care hospital in Kathmandu, Nepal. The Canadian Journal of Infectious Diseases \& Medical Microbiology. 2017; 2017:2191532. [DOI:10.1155/2017/2191532] [PMID] [PMCID]

9. Ghasabi F, Halaji M, Nouri S. Determination of vancomycinresistant Staphylococcus aureus. Journal of Research in Pharmacy Practice. 2017; 6(1):60. [DOI:10.4103/2279042X.200986] [PMID] [PMCID]

10. O'Hara FP, Suaya JA, Ray GT, Baxter R, Brown ML, Mera $\mathrm{RM}$, et al. Spa typing and multilocus sequence typing show comparable performance in a macroepidemiologic study of Staphylococcus aureus in the United States. Microbial Drug Resistance. 2016; 22(1):88-96. [DOI:10.1089/ mdr.2014.0238] [PMID] [PMCID]

11. Askari E, Zarifian A, Pourmand M, Naderi-Nasab M. Highlevel vancomycin-Resistant Staphylococcus aureus (VRSA) in Iran: A systematic review. Journal of Medical Bacteriology. 2012; 1(3-4):53-61.

12. Rezai MS, Pourmousa R, Dadashzadeh R, Ahangarkani F. Multidrug resistance pattern of bacterial agents isolated from patient with chronic sinusitis. Caspian Journal of Internal Medicine. 2016; 7(2):114-9.

13. Shekarabi $M$, Hajikhani B, Salimi Chirani A, Fazeli $M$, Goudarzi M. Molecular characterization of vancomycin-resistant Staphylococcus aureus strains isolated from clinical samples: A three year study in Tehran, Iran. PLOS One. 2017; 12(8):e0183607. [DOI:10.1371/journal. pone.0183607] [PMID] [PMCID]

14. Guven Gokmen T, Kalayci Y, Yaman A, Koksal F. Molecular characterization of methicillin-resistant Staphylococcus aureus strains by spa typing and pulsed field gel electrophoresis methods. BMC Microbiology. 2018; 18:155. [DOI:10.1186/s12866-018-1305-6] [PMID] [PMCID] 
15. CLSI. Performance standards for antimicrobial susceptibility testing, $27^{\text {th }}$ ed . Philadelphia. Clinical and Laboratory Standards Institute; 2017.

16. McGuinness WA, Malachowa N, DeLeo FR. Vancomycin resistance in Staphylococcus aureus. The Yale Journal of Biology and Medicine. 2017; 90(2):269-81.

17. Struelens MJ. Consensus guidelines for appropriate use and evaluation of microbial epidemiologic typing systems. Clinical Microbiology and Infection. 1996; 2(1):2-11. [DOI:10.1111/j.1469-0691.1996.tb00193.x] [PMID]

18. Wertheim HF, Melles DC, Vos MC, van Leeuwen W, van Belkum A, Verbrugh HA, et al. The role of nasal carriage in Staphylococcus aureus infections. The Lancet Infectious Diseases. 2005; 5(12):751-62. [DOI:10.1016/S14733099(05)70295-4]

19. Behzadnia S, Davoudi A, Rezai MS, Ahangarkani F. Nosocomial infections in pediatric population and antibiotic resistance of the causative organisms in North of Iran. Iranian Red Crescent Medical Journal. 2014; 16(2):e14562. [DOI:10.5812/ircmj.14562] [PMID] [PMCID]

20. Afshari SG, Sepahi AA, Goudarzi H, Tabrizi MS, Goudarzi M, Hajikhani B, et al. Distribution of SCCmec types in methicillin-resistant Staphylococcus aureus isolated from burn patients. Archives of Clinical Infectious Diseases. 2017; 12(2):e62760. [DOI:10.5812/archcid.62760]

21. Navaeifar MR, Rezai MS. Device associated nosocomial infection in children. Journal of Pediatrics Review. 2013; 1(2):25-41.

22. Tavares DA, Sa-Leao R, Miragaia $M$, de Lencastre $H$. Large screening of CA-MRSA among Staphylococcus aureus colonizing healthy young children living in two areas (urban and rural) of Portugal. BMC Infectious Diseases. 2010; 10:110. [DOI:10.1186/1471-2334-10-110] [PMID] [PMCID]

23. Rahimzadeh G, Gill P, Rezai MS. Ultra structural characteristics of methicillin resistant Staphylococcus aureus cell wall after affecting with lytic bacteriophages using atomic force microscopy. Iranian Journal of Basic Medical Sciences. 2019; 22(3):290-5. [DOI:10.22038/IJBMS.2019.31226.7521]

24. Rahimzadeh G, Gill P, Rezai MS. Characterization of Methicillin-Resistant Staphylococcus Aureus (MRSA) phages from sewage at a tertiary pediatric hospital. Archives of Pediatric Infectious Diseases. 2017; 5(1):e39615. [DOI:10.5812/pedinfect.39615]

25. Rahimzadeh G, Gill P, Rezai MS. Endolysins of bacteriophages as an anti-methicillin resistant staphylococcus aureus infection in children: A narrative review. Journal of Pediatrics Review. 2018; 6(1):36-43. [DOI:10.5812/jpr.11562]

26. Goudarzi M, Fazeli M, Goudarzi H, Azad M, Seyedjavadi SS. Spa typing of staphylococcus aureus strains isolated from clinical specimens of patients with nosocomial infections in Tehran, Iran. Jundishapur Journal of Microbiology. 2016; 9(7):e35685. [DOI:10.5812/jjm.35685]
27. Rezai MS, Shahmohammadi S. Nosocomial Infections in Iranian pediatric patients with burn injuries: A review. Journal of Pediatrics Review. 2015; 3(2):1-11. [DOI:10.17795/ jpr-680]

28. Chen JH, Cheng VC, Chan JF, She KK, Yan MK, Yau MC, et al. The use of high-resolution melting analysis for rapid spa typing on methicillin-resistant Staphylococcus aureus clinical isolates. Journal of Microbiological Methods. 2013; 92(2):99-102. [DOI:10.1016/j.mimet.2012.11.006] [PMID]

29. Fasihi Y, Fooladi S, Mohammadi MA, Emaneini M, Kalantar-Neyestanaki D. The spa typing of methicillin-resistant Staphylococcus aureus isolates by High Resolution Melting (HRM) analysis. Journal of Medical Microbiology. 2017; 66(9):1335-7. [DOI:10.1099/jmm.0.000574]

30. Mazi W, Sangal V, Sandstrom G, Saeed A, Yu J. Evaluation of spa-typing of methicillin-resistant Staphylococcus aureus using high-resolution melting analysis. International Journal of Infectious Diseases. 2015; 38:125-8. [DOI:10.1016/j. ijid.2015.05.002] [PMID] 